\title{
Observation of Reversed-Shear Alfven Eigenmodes Excited by Energetic lons in a Helical Plasma
}

\section{AUTHOR(S):}

Toi, K.; Watanabe, F.; Tokuzawa, T.; Ida, K.; Morita, S.; Ido, T.; Shimizu, A.; ... Yoshinuma, M.; Kawahata, K.; Komori, A.

\section{CITATION:}

Toi, K.... [et al]. Observation of Reversed-Shear Alfven Eigenmodes Excited by Energetic Ions in a Helical Plasma. Physical Review Letters 2010, 105(14): 145003.

\section{ISSUE DATE:}

2010

URL:

http://hdl.handle.net/2433/148388

RIGHT:

(C) 2010 The American Physical Society 


\title{
Observation of Reversed-Shear Alfvén Eigenmodes Excited by Energetic Ions in a Helical Plasma
}

\author{
K. Toi, ${ }^{1}$ F. Watanabe, ${ }^{2}$ T. Tokuzawa,,${ }^{1}$ K. Ida, ${ }^{1}$ S. Morita, ${ }^{1}$ T. Ido, ${ }^{1}$ A. Shimizu, ${ }^{1}$ M. Isobe,${ }^{1}$ K. Ogawa, ${ }^{2}$ D. A. Spong, ${ }^{3}$ \\ Y. Todo, ${ }_{1}^{1}$ T. Watari, ${ }^{1}$ S. Ohdachi, ${ }^{1}$ S. Sakakibara, ${ }^{1}$ S. Yamamoto, ${ }^{4}$ S. Inagaki, ${ }^{5}$ K. Narihara, ${ }_{1}^{1}$ M. Osakabe, ${ }^{1}$ K. Nagaoka, ${ }^{1}$ \\ Y. Narushima, ${ }^{1}$ K. Y. Watanabe, ${ }^{1}$ H. Funaba, ${ }^{1}$ M. Goto, ${ }^{1}$ K. Ikeda, ${ }^{1}$ T. Ito, ${ }^{2}$ O. Kaneko, ${ }^{1}$ S. Kubo, ${ }^{1}$ S. Murakami, ${ }^{4}$ \\ T. Minami, ${ }^{1}$ J. Miyazawa, ${ }^{1}$ Y. Nagayama, ${ }^{1}$ M. Nishiura, ${ }^{1}$ Y. Oka, ${ }^{1}$ R. Sakamoto,${ }^{1}$ T. Shimozuma,${ }^{1}$ Y. Takeiri, ${ }^{1}$ K. Tanaka, ${ }^{1}$ \\ K. Tsumori, ${ }^{1}$ I. Yamada, ${ }^{1}$ M. Yoshinuma, ${ }^{1}$ K. Kawahata, ${ }^{1}$ A. Komori, ${ }^{1}$ and LHD Experiment Group ${ }^{1}$ \\ ${ }^{1}$ National Institute for Fusion Science, Toki, Japan \\ ${ }^{2}$ Department of Energy Engineering and Science, Nagoya University, Nagoya, Japan \\ ${ }^{3}$ Oak Ridge National Laboratory, Oak Ridge, Tennessee, USA \\ ${ }^{4}$ Kyoto University, Kyoto, Japan \\ ${ }^{5}$ Research Institute for Applied Mechanics, Kyusyu University, Kasuga, Japan
}

(Received 27 September 2008; published 1 October 2010)

\begin{abstract}
Reversed-shear Alfvén eigenmodes were observed for the first time in a helical plasma having negative $q_{0}^{\prime \prime}$ (the curvature of the safety factor $q$ at the zero shear layer). The frequency is swept downward and upward sequentially via the time variation in the maximum of $q$. The eigenmodes calculated by ideal MHD theory are consistent with the experimental data. The frequency sweeping is mainly determined by the effects of energetic ions and the bulk pressure gradient. Coupling of reversed-shear Alfvén eigenmodes with energetic ion driven geodesic acoustic modes generates a multitude of frequency-sweeping modes.

PACS numbers: 52.35.Bj, 52.35.Mw, 52.55.Hc, 52.55.Pi
\end{abstract}

Alfvén eigenmodes are investigated in many tokamaks because they can be destabilized by energetic alphas and beam ions in magnetically confined fusion plasmas. In particular, the discovery of reversed-shear Alfvén eigenmodes (RSAEs) or Alfvén cascades [1-4] has generated a great deal of attention because these modes are excited in reversed magnetic shear (RS) configurations of tokamaks, which are attractive for internal transport barrier formation. The frequency of the RSAEs typically is swept upward to the toroidal Alfvén eigenmode frequency as the local minimum of the safety factor $q_{\min }$ drops in time. RSAEs can exist just above the maxima or just below the minima of shear Alfvén continua and are similar to the global Alfvén eigenmode. The RSAE localizes around the maxima or minima of the continua, while the global Alfvén eigenmode has a radially extended structure [5]; both are typically dominated by a single Fourier mode. The frequency sweeping of RSAE is explained by introducing several factors $[1,6-9]$ such as energetic particles and a pressure gradient. The finite minimum of the RSAE frequency, observed at the rational values of $q_{\min }=m / n$ ( $m$ and $n$ are poloidal and toroidal mode numbers, respectively), depends on the geodesic acoustic mode (GAM) frequency [10] and the frequency offset due to the gradients of energetic ions and bulk plasma pressure [11]. This means that the shear Alfvén perturbations are no longer incompressible around the minimum frequency. The time evolution of the RSAE frequency can then be employed as a useful diagnostic tool. It is of importance to compare characteristics of Alfvén eigenmodes (AEs) in helical and tokamak plasmas with RS configurations.
In the Large Helical Device (LHD) [12], an RS configuration, characterized by a nonmonotonic rotational transform $(\iota / 2 \pi)$ profile, was obtained in low density plasmas with intense counter neutral beam current drive (NBCD). The counter NBCD induces plasma current to reduce the externally generated rotational transform. The discharge waveform is shown in Fig. 1(a), where the magnetic axis position in the vacuum field is $R_{\mathrm{ax}}=3.75 \mathrm{~m}$ and the toroidal field $B_{t}=1.3 \mathrm{~T}$. The line averaged electron density is raised by neon gas puffing into the hydrogen target plasma to minimize shinethrough of the injected neutral beams and electron return current in NBCD. The effective charge of the plasma in the constant density phase $(2 \mathrm{~s}<$ $t<4 \mathrm{~s}$ ) is estimated to be $Z_{\text {eff }} \approx 6$. A parabolic electron temperature profile of $T_{\mathrm{eo}} \sim 1.2 \mathrm{keV}$ and hollow density profile of $n_{\mathrm{eo}} \sim 0.4 \times 10^{19} \mathrm{~m}^{-3}$ were sustained in the phase. The central ion temperature $T_{\text {io }}$ was $\sim 0.8 \mathrm{keV}$ in the phase. Here, counter NBCD with $\sim 2.2 \mathrm{MW}$ and beam energy $E_{b} \sim 160 \mathrm{keV}$ was carried out until $t=4 \mathrm{~s}$ and then stepped down to $\sim 1.6 \mathrm{MW}$. In this shot, the $\iota / 2 \pi$ profile was measured by motional stark effect spectroscopy. The measured profiles are shown in Fig. 1(b), together with two profiles interpolated from these data, where $\rho$ is the normalized minor radius. The $\iota / 2 \pi$ profile evolves in time, having an off-axis minimum of $\iota / 2 \pi$ $\left[(\iota / 2 \pi)_{\min }\right]$. This clearly indicates the formation of a RS configuration in helical plasma. The curvature of the safety factor $q[=1 /(\iota / 2 \pi)]$ is $q_{0}^{\prime \prime}<0$ at the zero shear layer $r_{o}$, in contrast to $q_{0}^{\prime \prime}>0$ in the RS tokamak. A typical spectrogram of the magnetic probe signal in this shot is shown in Fig. 2(a). The mode number $n$ is determined by 5 probes 


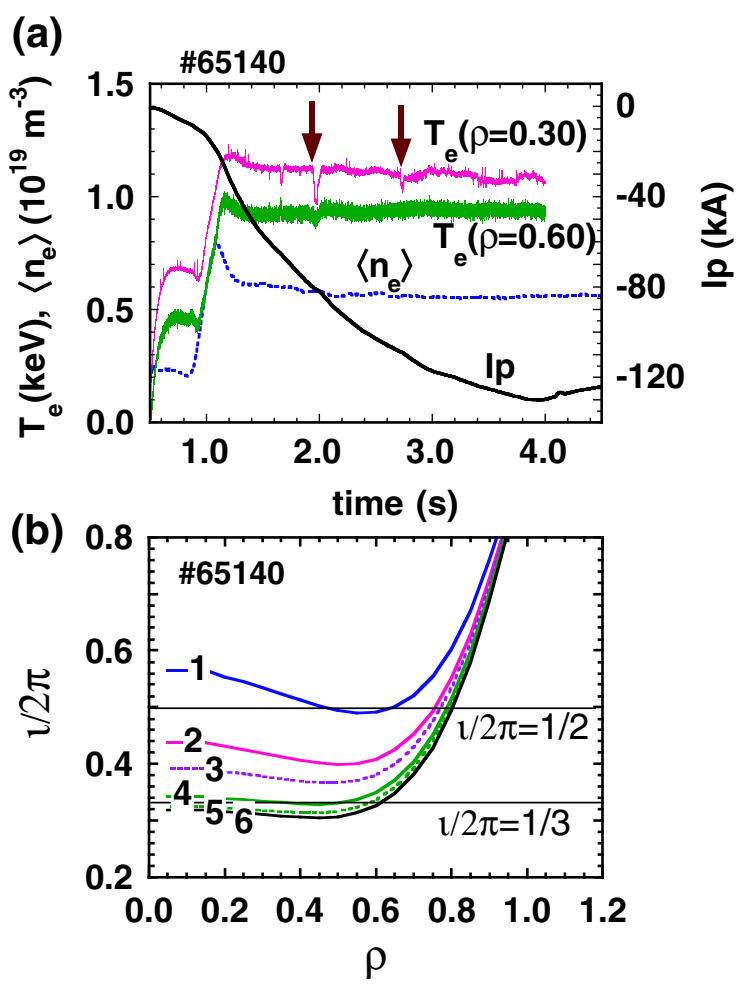

FIG. 1 (color online). (a) Time evolution of line averaged electron density $\left\langle n_{e}\right\rangle$; electron temperatures at $\rho \sim 0.3$ and $\sim 0.6$ measured by ECE polychromator and plasma current $I_{p}$. (b) The $\iota / 2 \pi$ profiles derived from motional stark effect spectroscopy at $t=1.8$ (curve 1), 2.3 (2), 2.8 (4), and $3.3 \mathrm{~s} \mathrm{(6).} \mathrm{The} \mathrm{profiles}$ interpolated in time are also shown: $t=2.5$ (curve 3 ) and $3.0 \mathrm{~s}(5)$.

arranged in the toroidal direction, and $m$ by 13 probes arranged poloidally. Two types of coherent modes are clearly identified in the constant density phase. As indicated by arrows, one is an $n=1$ mode exhibiting a characteristic downward-to-upward frequency sweeping via the minimum values at $t \sim 2 \mathrm{~s}$ and $t \sim 2.75 \mathrm{~s}$. At these time slices, the electron temperature at $\rho \sim 0.3$ has a short dip, as shown in Fig. 1(a). The $n=1$ mode is dominated by $m \sim 2$ in the former sweeping phase until $t \sim 2.4 \mathrm{~s}$, and then the $n=1$ mode is dominated by $m \sim 3$ in the latter phase. The characteristic time evolution of the $n=1$ mode has a similarity to that of the RSAE in the RS tokamak, except for the symmetric (downward-to-upward) sweeping. The remaining mode is a peculiar mode having $n=0$. The mode appears from $t \sim 1.8 \mathrm{~s}$ and gradually grows with the decrease of $(\iota / 2 \pi)_{\min }$ in time, having almost constant frequency. These mode activities are also detected by other fluctuation diagnostics: microwave interferometer and reflectometer, electron cyclotron emission (ECE) polychromator, and so on. Figure 2(b) shows the spectrogram of the signal obtained by the microwave interferometer along the central chord. The interferometer signal does not represent local density fluctuations, but it reflects density fluctuations across the whole plasma region and thereby provides information on the frequencies of various coherent modes. The spectrogram shows many coherent modes present with

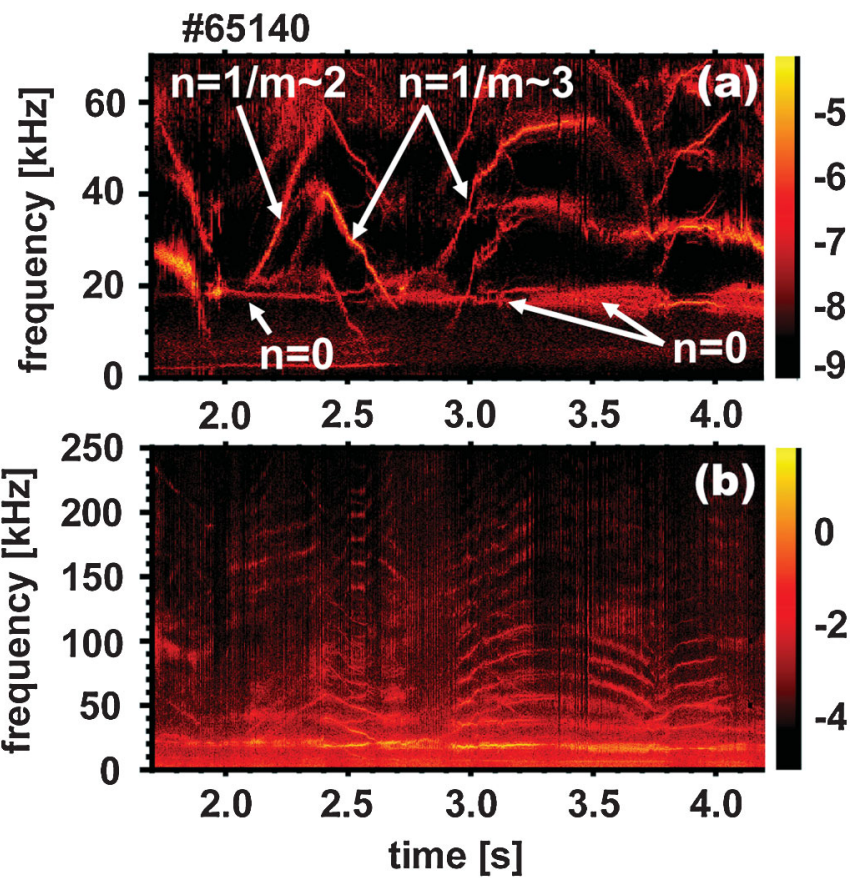

FIG. 2 (color). Spectrograms of magnetic probe signal $\left(d b_{\theta} / d t\right)$ (a) and microwave interferometer signal $\left(I_{\mu}\right)$ taken along the central chord (b). Color codes, respectively, indicate $\log _{10}\left[\left(d b_{\theta} / d t\right)^{2}\right]$ in (a) and $\log _{10}\left[I_{\mu}^{2}\right]$ in (b).

frequencies equally shifted by the frequency of $n=0$ mode and higher harmonics of the $n=0$ mode.

Shear Alfvén spectra were calculated for two time slices at $t=2.5 \mathrm{~s}$ and $t=3.0 \mathrm{~s}$, by using the experimental data shown in Fig. 1. The time $t=2.5 \mathrm{~s}$ is in the phase where the $n=1$ mode frequency is swept downward. The time $t=3.0 \mathrm{~s}$ is in the upward sweeping phase. Figure 3(a) shows the shear Alfvén spectra at $t=2.5 \mathrm{~s}$ and $t=$ $3.0 \mathrm{~s}$, where the hydrogen mass density was multiplied by 1.5 times because of neon-doped plasma. AEs were searched in the shear Alfvén spectra by the AE3D code, which is based on the ideal MHD theory for low beta and incompressible plasma [13] and retains the threedimensional variation of the stellarator equilibrium. The expected eigenmodes relevant to the observed $n=1$ mode were found, and the frequency and radial extent of the eigenfunctions is shown by two horizontal bars (1) and (2) in Fig. 3(a). The calculated eigenfrequencies are, respectively, $37.91 \mathrm{kHz}$ for mode (1) and $27.85 \mathrm{kHz}$ for mode (2). For them, the observed mode frequencies are 33 and $36 \mathrm{kHz}$, respectively, where the Doppler shift effect due to toroidal plasma rotation can be neglected as a result of low toroidal rotation velocity $<5 \mathrm{~km} / \mathrm{s}$. The eigenfrequencies calculated for other time slices follow the frequency sweeping observed with the decrease in $(\iota / 2 \pi)_{\min }$ very well, except near the minimum frequency $(t \sim 2.75 \mathrm{~s})$. Here, toroidal mode coupling was neglected because the mode coupling is unimportant for the RSAE modes. This code can be applied to analyze AEs, except for cases of rational values of $(\iota / 2 \pi)_{\min }$. The eigenfunctions of the 

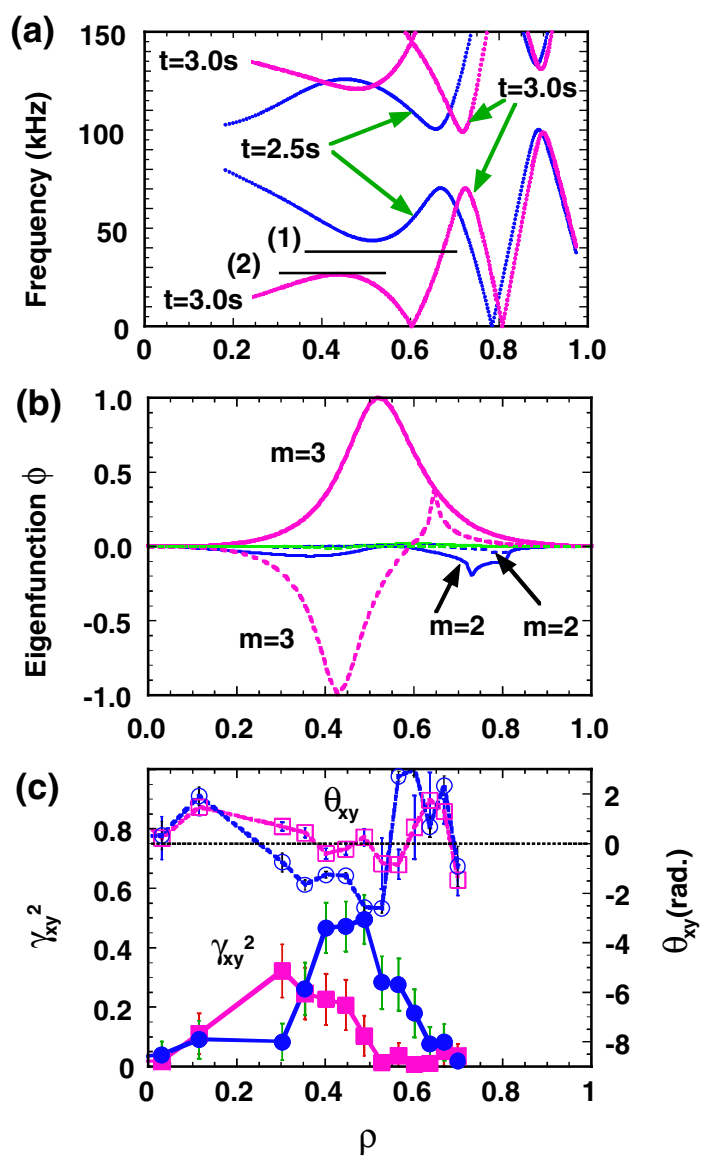

FIG. 3 (color online). (a) $n=1$ shear Alfvén spectra calculated by AE3D code at $t=2.5$ and $3.0 \mathrm{~s}$. (b) Eigenfunctions of RSAEs calculated by AE3D code, at $t=2.5 \mathrm{~s}$ (solid curves) and $t=3.0 \mathrm{~s}$ (broken curves). (c) Radial profiles of coherence and phase between ECE and magnetic probe signals in the window of $t=2.52-2.56 \mathrm{~s}$. Solid and open circles denote RSAEs and rectangles GAM.

electrostatic potential $\phi$ for these modes are shown in Fig. 3(b). The eigenfunctions are dominantly composed with a single Fourier mode $m=3 / n=1$ and relatively narrow. The information on the radial structure of $n=1$ mode was obtained from the ECE data. It is inferred from the radial profiles of coherence $\gamma_{x y}{ }^{2}$ and phase $\theta_{x y}$ between the ECE signals of each channel and the magnetic probe signal. Thus derived $\gamma_{x y}{ }^{2}$ and $\theta_{x y}$ profiles at $t=2.5 \mathrm{~s}$ are shown in Fig. 3(c), where 37 ensemble averages were done for each $39 \mathrm{~ms}$ time window. The AE temperature fluctuation due to a plasma displacement $\xi$ is expressed as $\delta T_{e} / T_{e}=-(\gamma-1) \boldsymbol{\nabla} \cdot \boldsymbol{\xi}-\boldsymbol{\xi} \cdot \boldsymbol{\nabla} T_{e} / T_{e}$, where $\gamma$ is the specific heat. If the $n=1$ mode produces only incompressible perturbations, then the second term of the right-hand side is dominant. The radial displacement $\xi_{r}$ due to an $\mathrm{AE}$ is approximately expressed as $\xi_{r} \approx[(\delta \mathbf{E} \times \mathbf{B}) /$ $\left.\left(\omega B^{2}\right)\right]_{r} \approx-m(\phi / r) /(\omega B)$. The radial structure of $\xi_{r}$ is approximately proportional to $\phi / r$. The eigenfunction at $t=2.5 \mathrm{~s}$ has a peak at $\rho \sim 0.5$ and has a similar radial extent to that of $\gamma_{x y}{ }^{2}$ profile shown in Fig. 3(c).
The fluctuation level of the $n=1$ mode at $t=3.0 \mathrm{~s}$ is fairly weak but has still a clear peak at $\rho \sim 0.45$. The results of AE3D are consistent with these experimental data. In addition, $\gamma_{x y}^{2}$ and $\theta_{x y}$ for the $n=0$ mode are also shown in Fig. 3(c). The $n=0$ mode locates in the plasma core region. In addition, observed density fluctuations of $n=0$ mode dominantly come from plasma compressibility $(\nabla \cdot \boldsymbol{\xi} \neq 0)$ in the core region where $\nabla n_{e}$ is very small, because the fluctuation is expressed as $\delta n_{e} / n_{e}=-\boldsymbol{\nabla} \cdot \boldsymbol{\xi}-\boldsymbol{\xi} \cdot \boldsymbol{\nabla} n_{e} / n_{e}$. Moreover, the mode frequency is decreased (increased) with the decrease (increase) in $T_{e}$ by cold gas injection (electron heating). The $n=0$ mode observed in this NBCD plasma is thought to be the GAM [14] excited by energetic ions [15-17], of which driving free energy is associated with the velocity space gradients. In a helical such as LHD, energetic beam ion loss and large orbit widths would in some situations create positive velocity gradients in the distribution function. The GAM frequency is given by the kinetic theory for helical plasmas $[18,19]$ as $f_{\mathrm{GAM}}=$ $C_{\text {hel }} \sqrt{\left[T_{e}+(7 / 4) T_{i}\right] /\left(C_{z} m_{i}\right)} \sqrt{2\left[1+(\iota / 2 \pi)^{2} F\right]} /(2 \pi R)$, where $F$ and $C_{\text {hel }}$, respectively, denote order unity correction factors dependent on $T_{e} / T_{i}$ and helical field ripple. The factor $C_{z}$ stands for the enhancement factor of mass density for hydrogen mass $\left(m_{i}\right)$ due to neon. The frequency calculated with $C_{\text {hel }}=1$ and $C_{z}=1.5$ is $f_{\mathrm{GAM}}=20 \mathrm{kHz}$ and is close to the observed frequency $f \approx 19 \mathrm{kHz}$.

We investigate the requirement for energetic ion content which would lead to the characteristic frequency sweeping in RSAEs. The existence criterion of RSAEs for the RS tokamak will be applicable for this analysis, because the 3D effects are not important for RSAEs. The existence is evaluated by the potential well function $Q$ in the eigenmode equation, that is, $Q>1 / 4$ [1,7-10]. The $Q$ consists of four main contributions: energetic ion content $\left(Q_{h}\right)$ [1], toroidicity effect $\left(Q_{t}\right)$ [7], plasma pressure gradient $\left(Q_{p}\right)$ [9], and electron density gradient $\left(Q_{d}\right)$ [8]. For high $m$ mode in a large aspect ratio tokamak, $Q_{p}$ is expressed as $Q_{p} \approx \frac{m}{\left(m-n q_{0}\right)}\left[\bar{\kappa}_{r} \alpha q_{0} /\left(r_{o}^{2} q_{0}^{\prime \prime}\right)\right]_{r=r_{o}}$ [9], where $\bar{\kappa}_{r}$ is the fluxsurface averaged normal curvature and $\alpha=-R_{o} q_{0}^{2} d \beta / d r$ is the normalized pressure gradient $\left[R_{o}\right.$ is the major plasma radius and $\beta=$ (bulk pressure)/(magnetic pressure)]. In this plasma, $\bar{\kappa}_{r}$ and $\alpha$ are positive, because of the magnetic well at the zero shear layer. Energetic beam ions of $E_{b} \sim 160-180 \mathrm{keV}$ at $B_{t}=1.3 \mathrm{~T}$ deviate appreciably from the magnetic surface in this plasma $(\Delta / a \sim$ $0.2-0.3 ; \Delta$ is the orbit deviation). For an approximate evaluation of the energetic ion contribution, we use $Q_{h}$ derived in large orbit limit [1]:

$$
\begin{aligned}
Q_{h} \approx & \pm \frac{R_{o} q_{0} \omega_{\mathrm{ch}}}{V_{A}\left(r_{o}\right)} \frac{\left(m-n q_{0}\right)}{\left|m-n q_{0}\right|} \frac{q_{0}}{r_{o}^{2} q_{0}^{\prime \prime}} \\
& \times\left[1 \pm \frac{V_{\| h}}{V_{A}} \frac{\left(m-n q_{0}\right)}{\left|m-n q_{0}\right|}\right]_{r=r_{o}}\left(-\frac{r_{o}}{\rho_{i}} \frac{d\left\langle\rho_{h}\right\rangle}{d r}\right)_{r=r_{o}} .
\end{aligned}
$$




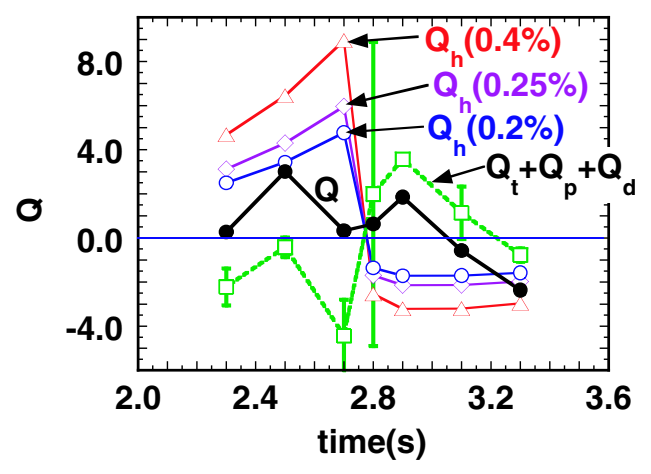

FIG. 4 (color online). (a) Time evolutions of $Q_{h}, Q_{t}+Q_{p}+$ $Q_{d}$, and $Q=Q_{h}+Q_{t}+Q_{p}+Q_{d}$. Thin solid curves indicate $Q_{h}$ for $\left\langle\beta_{h}\right\rangle=0.2 \%, 0.25 \%$, and $0.4 \%$. The solid curve indicates $Q$ for $\left\langle\beta_{h}\right\rangle=0.2 \%$, where $Q>1 / 4$ means the existence of RSAE.

Here, the fast ion driven current density is negative for the counter NBCD, and $V_{\| h} / V_{A} \approx-0.4$ at $r=r_{o}$. The mass densities of bulk plasma and fast ion are, respectively, expressed as $\rho_{i}$ and $\left\langle\rho_{h}\right\rangle$, where \langle\rangle stands for the flux-surface averaged value. The parameter $\omega_{\mathrm{ch}}$ stands for the cyclotron frequency of an energetic ion (proton). The lower sign in the $Q_{h}$ should be taken for the negative frequency wave. Note that the observed RSAE propagates in the electron diamagnetic drift direction, that is, the negative frequency wave. The values of $Q_{h}$ for the assumed amount of fast ions $\left\langle\beta_{h}\right\rangle$ are plotted together with $Q_{t}+$ $Q_{p}+Q_{d}$ in Fig. 4, where $\left\langle\beta_{h}\right\rangle \sim 0.20 \%$ corresponds to $\left[-\left(r_{o} / \rho_{i}\right)_{d}\left\langle\rho_{h}\right\rangle / d r\right]_{r=r_{o}}=0.016$ for the peaked radial profile of fast ions as $\left\langle\rho_{h}\right\rangle /\left\langle\rho_{h}\right\rangle(0)=\left(1-\rho^{2}\right)^{2}$. In the plasma, $Q_{p}$ is dominant among $Q_{t}, Q_{p}$, and $Q_{d}$. An asymmetric shape in $Q_{h}$ around $t=2.75 \mathrm{~s}$ is due to the counter NBCD effect and the negative frequency wave as observed in this plasma. The negative frequency wave may be excited by particle anisotropy in the phase space [20], because the calculated deposition profile of beam ions is centrally peaked due to low density plasma. For $\left\langle\beta_{h}\right\rangle \sim 0.20 \%$ and $0.25 \%$, both downward and upward frequency sweeping in RSAEs is possible around the time of $(\iota / 2 \pi)_{\min }=3$ (from $t \sim 2.5$ to $\sim 3.0 \mathrm{~s}$ ) where $Q>1 / 4$. Larger content of energetic ions as $\left\langle\beta_{h}\right\rangle \sim 0.4 \%$ would suppress the RSAE in the upward sweeping phase. Moreover, the minimum frequency of the RSAE is the sum of $f_{\mathrm{GAM}}$ and the frequency offset from the pressure gradient and fast ion response [11]. As seen from Fig. 2(a), the observed minimum frequency is almost the same or slightly lower than $f_{\mathrm{GAM}}$ (i.e., the $n=0$ mode frequency). The frequency offset is inferred to be zero or negative. From the condition, $\left[-\left(r_{o} / \rho_{i}\right) d\left\langle\rho_{h}\right\rangle / d r\right]_{r=r_{o}} \geq 0.010$ was obtained. This corresponds to $\left\langle\beta_{h}\right\rangle \geq 0.15 \%$. Based on the above simplified discussions, the observed symmetric sweeping in RSAE frequency is possible in the range of $\left\langle\beta_{h}\right\rangle$ from $\sim 0.15 \%$ to $\sim 0.25 \%$.

A multitude of the frequency-sweeping modes having $n=1$ is generated through coupling between $n=1 \mathrm{RSAE}$ and GAM $(n=0)$, as seen from Fig. 2. For instance, two downward frequency-sweeping $n=1$ modes of $f_{ \pm}=$ $f_{\mathrm{RSAE}} \pm f_{\mathrm{GAM}}$ are identified at $t=2.5 \mathrm{~s}$, that is, $f_{+}=$ $51 \mathrm{kHz}$ and $f_{-}=13 \mathrm{kHz}$, where $f_{\mathrm{RSAE}}=32 \mathrm{kHz}$ and $f_{\mathrm{GAM}}=19 \mathrm{kHz}$. The triplet of the RSAE, GAM, and the mode of $f_{+}$or $f_{-}$exactly satisfies the selection rules for frequency and toroidal mode number in the three-wave coupling process. The coupling was also confirmed through bicoherence analysis. This fact is also clearly seen in the interferometer signal [Fig. 2(b)].

In summary, RSAEs were identified for the first time in a helical plasma having $q_{0}^{\prime \prime}<0$ at the zero shear layer. The frequency and eigenfunction of the RSAE is consistent with ideal MHD theory. The sequential frequency sweeping of downward to upward is well explained by competitive effects of fast ions and the bulk pressure gradient. This research would contribute to fusion research in tokamaks.

The author (K. Toi) gratefully acknowledges fruitful discussions with B. N. Breizman, G. Y. Fu, and F. Zonca. This work is supported in part by the LHD project (NIFS07ULHH508) and the Grant-in-Aid for Scientific Research (No. 16082209 and No. 21360457).

[1] H. L. Berk et al., Phys. Rev. Lett. 87, 185002 (2001).

[2] S. E. Sharapov et al., Phys. Plasmas 9, 2027 (2002).

[3] M. Takechi et al., Phys. Plasmas 12, 082509 (2005).

[4] M. A. Van Zeeland et al., Nucl. Fusion 46, S880 (2006).

[5] T. E. Evans et al., Phys. Rev. Lett. 53, 1743 (1984).

[6] F. Zonca et al., Phys. Plasmas 9, 4939 (2002).

[7] B. N. Breizman et al., Phys. Plasmas 10, 3649 (2003).

[8] S. V. Konovalov et al., Phys. Plasmas 11, 2303 (2004).

[9] G. Y. Fu et al., Phys. Plasmas 13, 052502 (2006).

[10] B. N. Breizman et al., Phys. Plasmas 12, 112506 (2005).

[11] B. N. Breizman, in Theory of Fusion Plasmas, AIP Conf. Proc. No. 871 (AIP, New York, 2006).

[12] A. Iiyoshi et al., Nucl. Fusion 39, 1245 (1999).

[13] D. A. Spong et al., Phys. Plasmas 17, 022106 (2010).

[14] N. Winsor et al., Phys. Fluids 11, 2448 (1968).

[15] H. Berk et al., Nucl. Fusion 46, S888 (2006).

[16] G. Y. Fu, Phys. Rev. Lett. 101, 185002 (2008).

[17] R. Nazikian et al., Phys. Rev. Lett. 101, 185001 (2008).

[18] H. Sugama et al., Phys. Plasmas 13, 012501 (2006).

[19] T. Watari et al., Phys. Plasmas 13, 062504 (2006).

[20] H. V. Wong et al., Phys. Lett. A 251, 126 (1999). 